\title{
Clinical evaluation of panel testing by next- generation sequencing (NGS) for gene mutations in myeloid neoplasms
}

\author{
Chun Hang Au, Anna Wa, Dona N. Ho, Tsun Leung Chan and Edmond S. K. Ma*
}

\begin{abstract}
Background: Genomic techniques in recent years have allowed the identification of many mutated genes important in the pathogenesis of acute myeloid leukemia (AML). Together with cytogenetic aberrations, these gene mutations are powerful prognostic markers in AML and can be used to guide patient management, for example selection of optimal post-remission therapy. The mutated genes also hold promise as therapeutic targets themselves. We evaluated the applicability of a gene panel for the detection of AML mutations in a diagnostic molecular pathology laboratory.

Methods: Fifty patient samples comprising $46 \mathrm{AML}$ and 4 other myeloid neoplasms were accrued for the study. They consisted of 19 males and 31 females at a median age of 60 years (range: 18-88 years). A total of 54 genes (full coding exons of 15 genes and exonic hotspots of 39 genes) were targeted by 568 amplicons that ranged from 225 to $275 \mathrm{bp}$. The combined coverage was $141 \mathrm{~kb}$ in sequence length. Amplicon libraries were prepared by TruSight myeloid sequencing panel (Illumina, CA) and paired-end sequencing runs were performed on a MiSeq (Illumina) genome sequencer. Sequences obtained were analyzed by in-house bioinformatics pipeline, namely BWA-MEM, Samtools, GATK, Pindel, Ensembl Variant Effect Predictor and a novel algorithm ITDseek.

Results: The mean count of sequencing reads obtained per sample was 3.81 million and the mean sequencing depth was over 3000X. Seventy-seven mutations in 24 genes were detected in 37 of 50 samples (74 \%). On average, 2 mutations (range 1-5) were detected per positive sample. TP53 gene mutations were found in 3 out of 4 patients with complex and unfavorable cytogenetics. Comparing NGS results with that of conventional molecular testing showed a concordance rate of $95.5 \%$. After further resolution and application of a novel bioinformatics algorithm ITDseek to aid the detection of FLT3 internal tandem duplication (ITD), the concordance rate was revised to $98.2 \%$.
\end{abstract}

Conclusions: Gene panel testing by NGS approach was applicable for sensitive and accurate detection of actionable AML gene mutations in the clinical laboratory to individualize patient management. A novel algorithm ITDseek was presented that improved the detection of FLT3-ITD of varying length, position and at low allelic burden.

Keywords: Next-generation sequencing, Acute myeloid leukemia, Gene mutations, FLT3 internal tandem duplication, Cytogenetics, Bioinformatics

\footnotetext{
*Correspondence: eskma@hksh.com

Division of Molecular Pathology, Department of Pathology, 1/F Li Shu Fan Block, Hong Kong Sanatorium \& Hospital 2 Village Road, Happy Valley, Hong Kong, China
} 


\section{Background}

The molecular basis of AML is heterogeneous. Cytogenetic study is well documented as a mandatory test at diagnosis to stratify patients into favorable, intermediate and adverse prognostic categories [1]. The identification of gene mutations in AML allows the further characterization of the molecular heterogeneity of this disease, especially with the subgroup of intermediate risk AML that often exhibit a normal karyotype [2]. In the recommendation by the European LeukemiaNet (ELN), mutations involving three genes NPM1, FLT3 and CEBPA are considered in AML prognostication scheme [3]. Cytogenetically normal AML (CN-AML) with mutated NPM1 without FLT3-ITD, or mutated CEBPA, are incorporated in the favorable genetic group. Recent data shows that only double, but not single, CEBPA mutations confer a favorable prognosis [4-7]. Likewise the National Comprehensive Cancer Network (NCCN) Guideline for AML incorporates CN-AML with NPM1 mutation or isolated CEBPA mutation in the absence of FLT3-ITD in the better-risk status category, whilst CNAML with FLT3-ITD mutation is considered poor-risk [8]. The detection of concurrent KIT mutation relegates the core binding factor $(\mathrm{CBF})$-related $A M L$ from betterrisk to intermediate-risk.

With the sequencing of the AML genome [9], the first cancer genome to be sequenced, mutations involving many more genes important in leukemogenesis are being deciphered. Through the whole genome sequencing approach, the genomic and epigenomic landscapes of adult de novo AML [10] and the clonal architecture of secondary AML [11] are comprehensively described. The genetic aberrations can be grouped under nine categories defined according to biological function and a putative role in AML pathogenesis, namely transcription factor fusions, gene encoding nucleophosmin (NPM1), tumor suppressor genes, DNA methylation-related genes (DNMT3A, IDH1, IDH2 and TET2), activated signaling genes, chromatin modifying genes, myeloid transcription factor genes, cohesin complex genes and spliceosome complex genes [10]. The clinical utility of these AML gene mutations is under investigation [12, 13]. Besides serving as powerful prognostic indicators, the mutational profile may have the potential to affect treatment decision in AML. For example, patients in the favorable genetic subgroup of mutated NPM1 lacking FLT3-ITD may be considered for postremission chemotherapy alone without resorting to allogeneic bone marrow transplantation (BMT) [14], and more recently the most favorable treatment response is reported in mutated NPM1 lacking FLT3ITD and harboring IDH2 R140 mutation [15]. Regarding upfront treatment, DNMT3A and NPM1 mutations together with MLL translocations, predict for an improved outcome with high-dose daunorubicin induction chemotherapy [15]. Moreover, mutations of the DNA methylation genes may predict for response to hypomethylation agents [16], although this issue remains controversial [17]. Finally, these AML gene mutations are themselves novel drug targets, as evident by the clinical trials on the FLT3 inhibitors [18] and IDH2 inhibitors [19].

It should be noted that whole genome sequencing and whole exome sequencing are predominantly research tools. To serve clinical management needs, accurate genetic testing results should be reported with a reasonable short turnaround time. We perform clinical evaluation of a gene panel for testing myeloid disorders by NGS in a diagnostic molecular pathology laboratory setting.

\section{Methods \\ Patient samples}

Fifty patient samples were accrued from April 2011 to November 2014. They comprised 19 males and 31 females at a median age of 60 years (range 18-88 years). The diagnoses were $\mathrm{M} 0=1, \mathrm{M} 1=7, \mathrm{M} 2=12, \mathrm{M} 3=1$, M4 = 5, M5 = 3, M6 = 2, not otherwise specified (NOS) $=4$, AML with myelodysplasia (MDS) related changes $(\mathrm{AML}-\mathrm{TMDS})=5$, AML transformed from MDS or $\mathrm{MDS} / \mathrm{MPD}=3$, therapy-related $\mathrm{AML}=1$, refractory $\mathrm{AML}=2$, high-grade $\mathrm{MDS}=3$ and atypical chronic myeloid leukemia $=1$. A diagnosis of AML-NOS was rendered in four patients due to diagnosis on peripheral blood only $(\mathrm{n}=2)$, aparticulate bone marrow aspirate $(n=1)$ and diagnosis at another institution $(n=1)$. The study was approved by the Research Ethics Committee of Hong Kong Sanatorium \& Hospital (reference number: REC-2015-02).

DNA was extracted from the corresponding specimens (bone marrow $=42$ and peripheral blood $=8$ ) using QIAamp DNA Blood Mini Kit (Qiagen, Germany). Four additional samples were available from 3 patients for comparison (buccal swab and second peripheral blood samples of patient 31, posttreatment sample of patient 35 and relapsed sample of patient 36). Extracted DNA was quantified using Qubit dsDNA BR Assay Kit and Qubit 2.0 Fluorometer (Life Technologies, USA).

Any known mutation status of FLT3, NPM1, KIT, CALR, MPL, CSF3R by Sanger sequencing and JAK2 $\mathrm{V} 617 \mathrm{~F}$ by allele-specific polymerase chain reaction (PCR) and PCR-restriction fragment length polymorphism (PCR-RFLP) analysis of the patient samples were curated from the clinical record for comparison with NGS results. 


\section{Control samples}

Archival DNA samples $(n=11)$ with known mutations in CALR $(n=6)$, JAK2 $(n=2), \operatorname{KIT}(n=1), \operatorname{MYD} 88(n=$ $1)$ and TP53 $(n=1)$ were retrieved as positive controls to validate the NGS myeloid panel. DNA extracted from peripheral blood samples of healthy adults with normal complete blood profile $(n=18)$ were accrued in July and October 2014 as negative controls. These control samples were analyzed in the same way as the patient samples.

\section{NGS myeloid gene panel}

The myeloid gene panel targets 54 genes (full coding exons of 15 genes: BCOR, BCORL1, CDKN2A, CEBPA, CUX1, DNMT3A, ETV6/TEL, EZH2, KDM6A, IKZF1, PHF6, RAD21, RUNX1/AML1, STAG2 and ZRSR2, and exonic hotspots of 39 genes: $A B L 1, A S X L 1, A T R X, B R A F, C A L R, C B L, C B L B$, CBLC, CSF3R, FBXW7, FLT3, GATA1, GATA2, GNAS, HRAS, IDH1, IDH2, JAK2, JAK3, KIT, KRAS, KMT2A/MLL, MPL, MYD88, NOTCH1, NPM1, NRAS, PDGFRA, PTEN, PTPN11, SETBP1, SF3B1, SMC1A, SMC3, SRSF2, TET2, TP53, U2AF1 and WT1) by 568 amplicons (length range: 225-275 bp). The combined coverage was $141 \mathrm{~kb}$. Amplicon sequencing libraries were prepared from $50 \mathrm{ng}$ of DNA per sample using TruSight myeloid sequencing panel (Illumina, USA). A highly multiplexed pool of oligonucleotide pairs upstream and downstream to each region of interest (ROI) was employed. Each oligonucleotide contained unique target-specific sequences and universal adaptor sequence used in the subsequent amplification reaction. For each sample, an extension-ligation reaction extended across the ROI and followed by ligation to unite the two probes to yield a library of templates with common ends. This library of new templates was PCR amplified with a unique pair of indexes incorporated for downstream sequence-based sample identification. After PCR clean-up, double-stranded DNA length and quantity of individual libraries were assessed by DNA 1000 kit and 2100 Bioanalyzer system (Agilent, USA). Libraries were normalized according to the measured quantity and pooled in batches ( 8 to 24 libraries per pool). Paired-end sequencing runs were performed on a MiSeq (Illumina, USA) with reagent kit v3 according to manufacturer's instructions.

\section{Variant calling and annotation}

Paired sequences obtained from each sample were mapped to human genome reference GRCh37/hg19 using BWA-MEM [20] version 0.7.7 with default parameters. Three variant callers were used: (1) Samtools [21] version 0.1.19, with mpileup command parameters $-\mathrm{L}$
100000 -d 100000 to cater for amplicons with depth exceeding 250-fold and bcftools command parameter$\mathrm{m} 0.99$ to use the new insertion-deletion (INDEL) calling model; (2) GATK HaplotypeCaller [22] version 2.8-1 according to the best practices recommended by the authors; and (3) VarScan [23] version 2.3.7 somatic mutation calling mode based on one of the negative controls or the matched germline DNA if available. For detection of FLT3 internal tandem duplication (ITD), additional variant callers were used specifically for the region chr13:28607161-28609590: (1) Pindel [24] version $0.2 .5 \mathrm{a} 7$ with the insert size configured as the summation of forward and reverse sequencing read length, to adapt the algorithm to the amplicon sequencing reads in this study, and (2) a novel algorithm ITDseek developed in this study (details described in a separate section).

Variant calls were first annotated by Ensembl Variant Effect [25] Predictor version 75 and then manually examined by at least two individuals. Sequence alignment of selected variants was manually examined with Integrative Genomics Viewer [26] (IGV). ROI sequencing depth was summarized using BEDTools [27] version 2.19.1 Minimum reportable variant allele frequency (AF) is $10 \%$ of sequencing depth at least 500 -fold. ROI with depth less than 500 -fold were regarded as sub-optimal regions. Variants found to be reported in COSMIC database version 67 and/or dbSNP version 138 were prioritized for manual examination while those reported in 1000 Genomes project (phase 1) were excluded. FLT3 ITD and ASXL1 c.1934dupG mutations were confirmed in selected patients using PCR fragment analysis by capillary electrophoresis (primer sequences available upon request) and analysis software Peak Scanner version 1.0 (http://www.appliedbiosystems.com). Variants were described according to the recommendations of Human Genome Variation Society (HGVS). Variant descriptions were checked by Mutalyzer Name Checker (http:// mutalyzer.nl).

\section{Evaluation of FLT3 ITD detection algorithms and development of a novel detection algorithm ITDseek} FLT3 ITD detection performance of Pindel, Samtools, GATK HaplotypeCaller were compared based on a simulated dataset of ITD mutations. An in silico FLT3 ITD sequencing read simulator ITDsim was developed based on BioPerl [28]. The amplicon targeting chr13:28,608,112-28,608,312 (both primer binding sites excluded) was chosen as the simulation target due to its highest reported FLT3 mutation rate in COSMIC among the three amplicons in the region. All combinations $(n=$ 40,401 ) of ITD lengths (range: $1-201 \mathrm{bp} ; n=201$ ) and starting positions (chr13:28,608,112-28,608,312; $n=201$ ) were simulated separately. ITD allelic burden was 
defined as $50 \%$. For each combination, 1000 paired-end ITD reads and 1000 paired-end wild-type reads (both $2 \times 275 \mathrm{bp}$ ) were simulated and the FASTQ file pair was subject to variant calling as described above. Simulation and corresponding variant calling were performed on a Cray XC30 supercomputer.

To overcome the difficulty in calling long ITD mutations with short-read NGS amplicon sequencing, a novel FLT3 ITD detection algorithm ITDseek was developed based on the following principles. It takes SAM/BAM alignments as input and outputs any detected ITD mutations in VCF format. In case of a short ITD (e.g., 9 bp) present in the middle of raw sequencing reads (e.g., $250 \mathrm{bp}$ ), the wild-type sequences upstream and downstream of ITD mutation are long enough for proper alignment to the FLT3 locus, with an insertion in between representing the additional sequence (operation "I" in CIGAR field of SAM alignment output by BWA-MEM). General-purpose variant callers like Samtools, GATK HaplotypeCaller and VarScan could then readily identify the inserted nucleotides. In contrast, if a given ITD is too long and/or too close to either end of amplicon, the sequence downstream of ITD mutation will become too short or even completely absent in the raw sequences obtained. Without long enough downstream sequences, the additional duplicated nucleotides are marked as soft-clipped instead (operation "S" in CIGAR field). These soft-clipped nucleotides are usually ignored by general-purpose variant callers as if they are sequencing adapter. In principle, realignment of soft-clipped nucleotides is a way to identify possible ITD mutations. Since BWA-MEM outputs a separate secondary alignment representing those softclipped nucleotides, such realignment is effectively performed. However, secondary alignments are usually ignored by general-purpose variant callers. ITDseek specifically searches for any soft-clipping points in primary alignments and correlate them with any corresponding secondary alignments for ITD identification. The length of ITD was extrapolated by the distance between the point of soft-clipping and the beginning of realignment of soft-clipped bases. The analysis is performed in individual reads and directions separately to identify any rare ITD clone (as rare as a single read only) or multiple ITD clones. In case of a special FLT3 ITD type that the additional sequence is entirely insertion of unknown origin, there is no secondary alignment. ITDseek identifies such ITD by comparing points of soft-clipping in sequencing read pairs. ITDseek was also evaluated based on the same simulated dataset described before. Source codes and documentation of ITDsim and ITDseek are available at http://github.com/tommyau/ITDseek for non-commercial use.

\section{Results}

High concordance of NGS results versus known mutations status

The NGS panel showed $100 \%$ concordance in detecting all 5 single-nucleotide mutations and 6 INDEL mutations from 11 corresponding positive control samples. The single-nucleotide mutations were JAK2 c.1849G > T p.Val617Phe $(n=2), \quad K I T \quad$ c.2447A $>$ T p.Asp816Val, MYD88 c.794 T >C p.Leu265Pro and TP53 c.916C > T p.Arg306* (all $n=1)$. The INDEL mutations included insertions of 5 bp (CALR c.1153_1154insCTTGT, c.1154_1155insTTGTC) and deletions of 19 bp (CALR c.1124_1142del), 34 bp (CALR c.1103_1136del) and 52 bp (CALR c.1099_1150del; $n=2)$. No mutation was detected from all 18 negative control samples.

Next, we compared NGS results with known mutation status of 42 patient samples from conventional testing ( $n=111$ results, both positive and negative), including FLT3 ITD $(n=39)$, FLT3 tyrosine kinase domain (TKD; $n=39)$, NPM1 $(n=22)$, JAK2 $(n=4), \operatorname{KIT}(n=4), C A L R$, $M P L$ and CSF3R (all $n=1$ ). The concordance rate was $95.5 \%$ (106 of 111), being observed in 17 mutation positive results (NPM1 $n=8$, FLT3 TKD $n=5$, FLT3 ITD $n$ $=3, K I T n=1)$ and 89 mutation negative results (FLT3 TKD $n=34$, FLT3 ITD $n=32$, NPM1 $n=14$, JAK2 and KIT both $n=3$, CALR, CSF3R and MPL all $n=1$ ). The 5 discrepancies were JAK2 V617F $(n=1)$ and FLT3 ITD $(n=4)$ mutations missed by NGS as elaborated below.

For patient 31, both allele-specific PCR and PCR-RFLP analysis of the initial sample (July 2014) showed JAK2 V617F weakly positive result (Fig. 1). No such mutation was reported by the NGS panel on the same sample. We asked whether the mutation was actually detected by

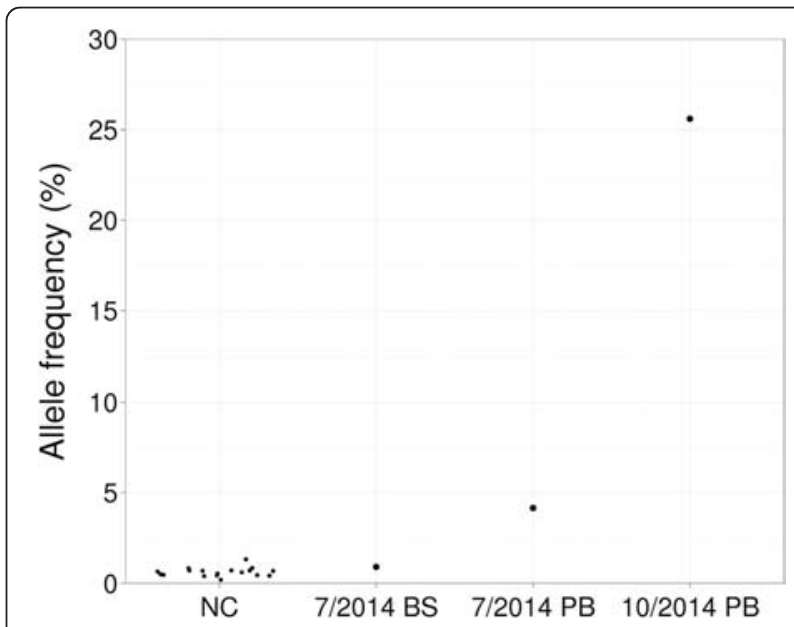

Fig. 1 JAK2 V617F allele frequencies as measured by next-generation sequencing. Samples included 18 negative controls (NC) and 3 samples of patient 31, namely buccal swab (BS) and peripheral blood (PB) samples in July 2014, and peripheral blood sample in October 2014. 
NGS but not reported based on the reportable minimum variant $\mathrm{AF}(10 \%)$. To obtain the AF of JAK2 V617 regardless of variant callers, we checked aligned reads at the corresponding genomic position chr9:5073770G $>\mathrm{T}$. V617F AF was $4.1 \%$ in the initial sample, $0.9 \%$ in matched buccal swab sample and $25.6 \%$ in the second peripheral blood sample (October 2014) (Fig. 1). The mean noise level was $0.6 \%$ based on 18 negative controls $(0.2-1.3 \%$, $99 \%$ confidence interval $0.45-0.79 \%$; Fig. 1). The slight but significant elevation of JAK2 V617F AF in the initial sample showed the mutation was indeed detectable by NGS and confirmed the weakly positive results by conventional molecular analysis.

Known FLT3 ITD mutations detected by Sanger sequencing in patients $3,36,37$ and 41 were originally not reported by the NGS panel. However, lengths of the ITD mutations (30-189 bp, $n=4)$ fell within the range reported to be detectable by Pindel (17-185 bp) [29], which was one of the variant callers used in this study. In the study by Spencer et al. employed sonication-based DNA fragmentation during sequencing library preparation, start positions of aligned reads were randomly distributed [29]. In contrast, start positions of aligned reads in this study were fixed by primers of corresponding PCR amplicons. We hypothesized that the ITD detection sensitivity also depended on the relative positions of ITD within corresponding amplicons.

\section{Comprehensive bioinformatics evaluation of FLT3 ITD detection methods}

We evaluated the performance of variant callers for ITD detection when the mutation position varied along the amplicon. A simulation approach was chosen to encompass a wide range of possible ITD mutations regardless of actual sample availability. A read simulator ITDsim was developed to simulate $2 \times 275$ bp sequencing data for ITD mutations at various starting positions, based on the amplicon with highest reported FLT3 ITD mutation rate (chr13:28,608,112-28,608,312) in the region. Given that the length of amplified region was $201 \mathrm{bp}$, starting positions included all these 201 positions and mutation length ranged from 1 to 201 bp (total 40401 combinations). For each combination of starting position and length, a pair of FASTQ files was simulated with sequencing depth 2000X and ITD AF $50 \%$. To determine the best possible performance of ITD detection, the simulated FLT3 sequencing reads were perfect-matching sequences derived from the GRCh37/hg19 reference genome without errors and all other amplicons were not simulated. Bioinformatics analysis of the simulated data was performed in the same way as the clinical samples.

Pindel, GATK HaplotypeCaller and Samtools detected $58.9,45.5$ and $23.0 \%$ of simulated ITD mutations, respectively (Fig. 2a). Although Pindel detected most ITD mutations among the three callers, the maximum length of detected ITD mutation decreased from 201 bp to $45 \mathrm{bp}$ at the $41^{\text {st }}$ nucleotide and $201^{\text {st }}$ nucleotide of the amplicon, respectively (Fig. 2a). ITD detection sensitivity was demonstrated to depend on not only length but also its relative position in the amplicon.

\section{A novel FLT3 ITD detection algorithm ITDseek}

We therefore closely examined the BWA-MEM alignments and observed two major differences between wild-type and ITD sequencing reads: (1) longer sequences became soft-clipped (ignored for analysis including variant calling), compared with consistently short soft-clipped sequences representing universal adapters, and (2) there was additional alignment of part of the soft-clipped sequences to the same FLT3 locus, but marked as supplementary alignment that was ignored for analysis in a similar manner as soft-clipping. Based on these observations, a novel ITD mutation algorithm ITDseek was developed and detected $73.1 \%$ of the simulated ITD mutations (Fig. 2a). For most starting positions in the amplicon (1 to 198), although the minimum length of detected ITD mutation ranged from 15 to $83 \mathrm{bp}$, the maximum detection length was consistently $201 \mathrm{bp}$ (the longest simulated length). ITDseek was insensitive to the remaining 3 starting positions (199-201 bp).

More importantly, ITDseek detected 96.8 \% (10152 of 10491) of combined false negatives by the other three callers (Fig. 2b). ITDseek increased overall detection rate from $74.0 \%$ (other three callers) to $99.2 \%$ (all four callers). Computation requirement of ITDseek was minimal that it took $<20 \mathrm{~s}$ and $<2 \mathrm{~GB}$ RAM to analyze a full MiSeq V3 run of 8 samples by 8 CPU cores (5-year-old Intel Xeon $2.8 \mathrm{GHz}$ processors).

After including ITDseek as an additional variant caller, known FLT3 ITD mutations of patients 3 (189 bp), 37 (30 bp) and 41 (30 bp) were detected to resolve 3 discrepancies. Regarding the remaining patient 36, ITDseek detected two different ITD mutations of lower AF (63 and $54 \mathrm{bp}$ ) but not the known 75 bp ITD mutation. A 3 bp deletion c.1739_1741delAGG located near the extension/ligation oligo binding site of amplicon 2 was also detected by the NGS panel (Fig. 3a). Reanalysis of original Sanger sequences confirmed the 3 bp deletion and suggested that it was in cis with 75 bp ITD mutation. Sanger sequencing of relapsed sample showed that the two in cis mutations became almost the only FLT3 allele detected. NGS of that relapsed sample detected the $3 \mathrm{bp}$ deletion at VAF $99.9 \%$. NGS sequencing depth of amplicon 2 dropped from 5500X (initial sample) to 70X (relapsed sample), which was consistent with complete drop-out of the ITD allele (Fig. 3a). PCR fragment 
analysis of the original and relapsed samples supported these observations (Fig. 3b).

In patient 20, which was reported ITD negative by Sanger sequencing, ITDseek detected two ITD mutations (119 and $25 \mathrm{bp}$ ). PCR fragment analysis confirmed both mutations (Fig. 3b). The allelic burden (1.4 and $3.2 \%$, respectively) was below the detection limit of Sanger sequencing. Taken together, after further resolution and application of ITDseek, there were still 2 samples (patients 20 and 36) with discrepancies and

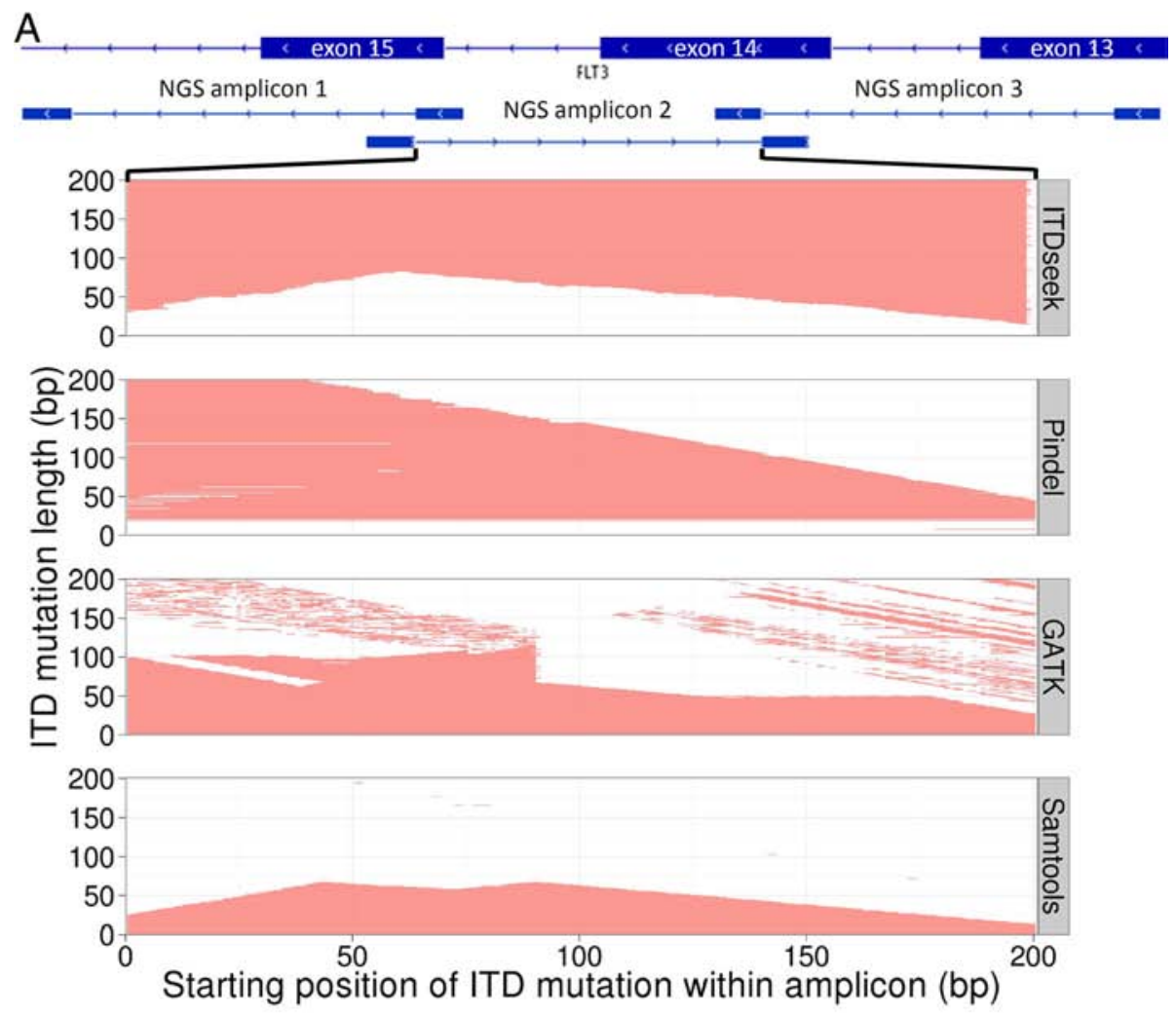

B

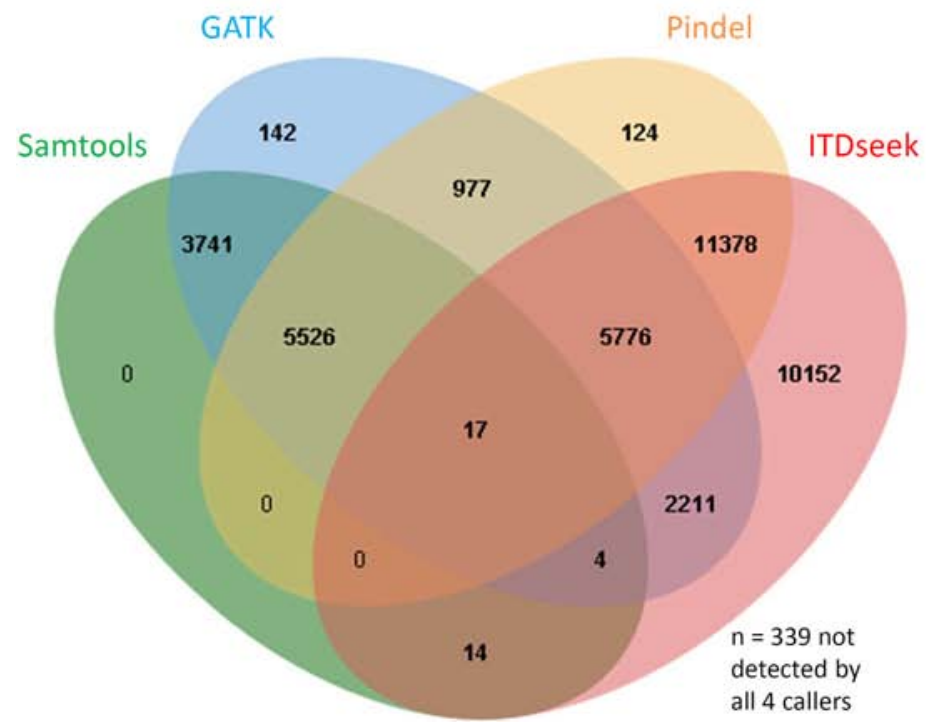

Fig. 2 FLT3 ITD detection performance of a novel algorithm ITDseek versus Pindel, GATK HaplotypeCaller and Samtools. a Detected combination of ITD length and relative position in the FLT3 NGS amplicon 2 (chr13:28,608,112-28,608,312) was indicated by red shading in the corresponding panel of each caller. $\mathbf{b}$ Venn diagram showing the number of ITD alleles detected by any or none of the four tested callers. 


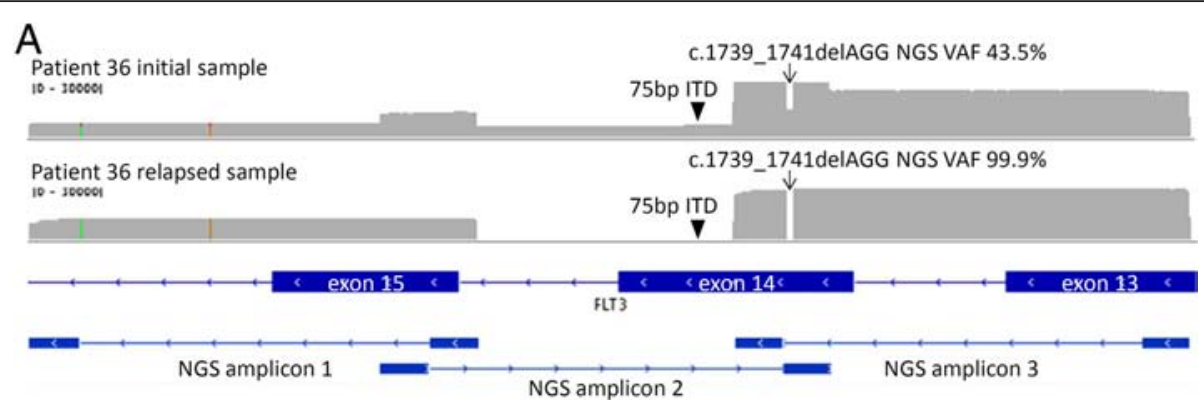

B

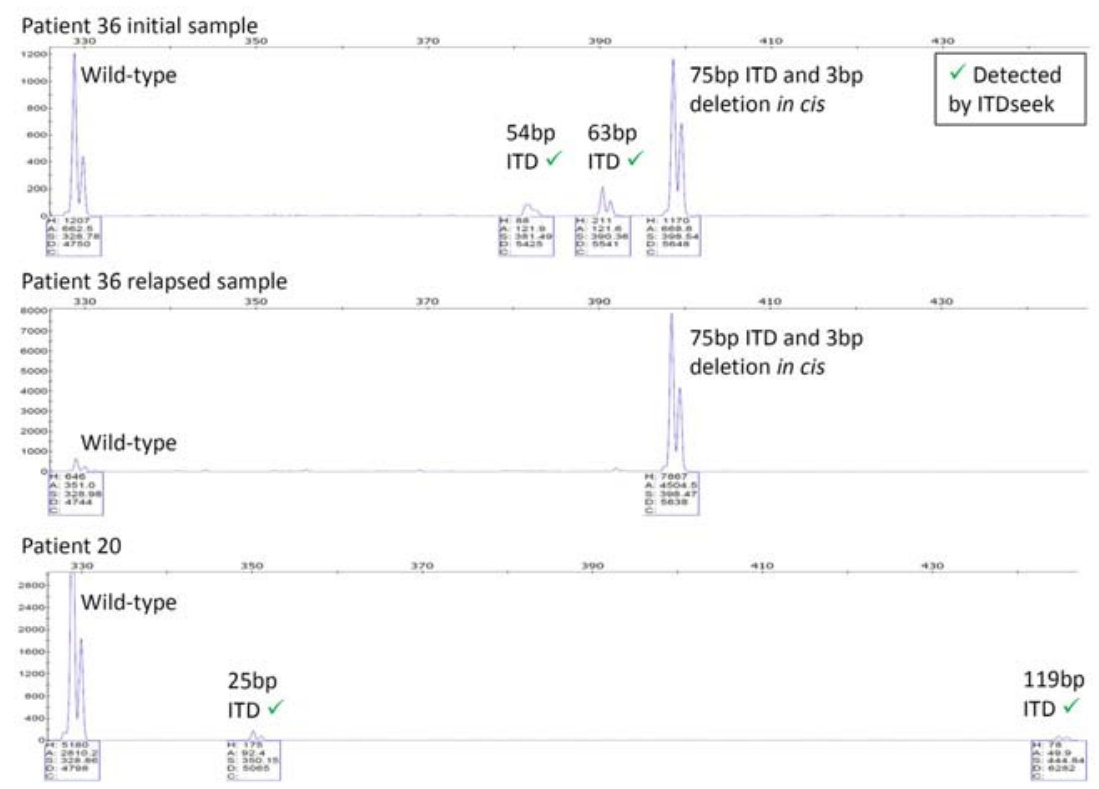

Fig. 3 FLT3 of patients 36 and 20 as characterized by next-generation sequencing and fragment analysis. a NGS sequencing depth histogram for 3 amplicons covering FLT3 exons 13 to 15 at scale 0-30000X. Magnitude of drops in sequencing depth at amplicon 2 and the region of 3 bp deletion (c.1739_1741delAGG; indicated by arrow) was proportional to the deletion VAF as indicated. The amplicon 2 covered the 75 bp ITD (indicated by triangle) but was affected by the 3 bp deletion in cis. b PCR fragment analysis for FLT3 ITD detection. ITD of 54 bp, 63 bp (patient 36 initial sample), 25 bp and 119 bp (patient 20) detected by NGS and ITDseek were confirmed by the corresponding fragments. Single additional fragment detected in both initial and relapsed samples of patient 36 confirmed the additional allele, which consisted of 75 bp ITD and 3 bp deletion in cis and was not detected by NGS due to allele drop-out.

concordance was $98.2 \%$ (109 of 111). FLT3 mutation detection was more sensitive by NGS since ITDseek detected 2 additional ITD alleles with low VAF in both patients 20 and 36, notwithstanding the one complex allele in patient 36 that was undetected by NGS due to allele drop-out.

\section{NGS mutation profile}

The mean count of sequencing reads obtained per sample was 3.81 million (range 2.02-8.51 million) and the mean sequencing depth was over 3000X. Seventy-seven mutations in 24 genes (Table 1) were detected in 37 of 50 patient samples (74\%). On average 2 mutations (range 1-5) were detected per positive sample. Mutations were detected in the following genes: FLT3 $(n=$ 16), NPM1 $(n=12), I D H 2(n=7), A S X L 1, D N M T 3 A$,
TET2, TP53 (all $n=4$ ), CEBPA, IDH1 (both $n=3$ ), CSF3R, KIT, RAD21, SMC3, STAG2 (all $n=2$ ), CBL, ETV6, EZH2, IKZF1, JAK2, NRAS, PTPN11, SETBP1, $S F 3 B 1$ and ZRSR2 (all $n=1$ ). The two most frequently mutated genes in our patient cohort, FLT3 and NPM1, were in keeping with the mutational frequency of genes in AML as reported in the literature (Fig. 4) [30]. Based on mechanism of action, genes involved in signal transduction (FLT3, CSF3R, KIT, NRAS, PTPN11, CBL and $J A K 2)$ and DNA methylation (DNMT3A, TET2, IDH1 and $I D H 2$ ) were the most frequent mutated groups, accounting for $24(31 \%)$ and $18(23 \%)$ out of 77 detectable mutations [31].

The small patient number in our cohort precluded meaningful correlation between gene mutations with clinical features and outcome or survival analysis. This 
Table 1 Summary of 50 patients in this study.

\begin{tabular}{|c|c|c|c|c|c|c|}
\hline No. & $\begin{array}{l}\text { Sex/ } \\
\text { Age }\end{array}$ & Diagnosis & Cytogenetics & Conventional molecular testing & NGS myeloid panel & Clinical outcome \\
\hline 1 & $M / 56$ & $\mathrm{AML}^{-\mathrm{NOS}^{*}}$ & Not done & FLT3- & KIT p.D816V & Alive on treatment \\
\hline 2 & $F / 37$ & M1 & $\begin{array}{l}\text { Near- } \\
\text { tetraploid and } \\
\text { complex }\end{array}$ & NPM-, FLT3- & TP53 p.E62 ${ }^{*}$ & Treated in Mainland China \\
\hline 3 & $\mathrm{M} / 60$ & $\begin{array}{l}\text { High grade } \\
\text { MDS }\end{array}$ & Normal & FLT3-ITD (189 bp), del(5q) FISH- & FLT3 ITD (189 bp) & Alive in CR after BMT \\
\hline 4 & $F / 83$ & M6 & No growth & FLT3-ITD-TKD+ & FLT3 p.D835Y & Died \\
\hline 5 & $M / 58$ & $\begin{array}{l}\text { High grade } \\
\text { MDS }\end{array}$ & $\begin{array}{l}\text { Complex }>3 \\
\text { abnormality }\end{array}$ & NPM1-, FLT3- & Negative & Died after BMT \\
\hline \multirow[t]{3}{*}{6} & \multirow[t]{3}{*}{$M / 57$} & \multirow[t]{3}{*}{ AML-TMDS } & \multirow[t]{3}{*}{ Normal } & \multirow[t]{3}{*}{ NPM1-, FLT3- } & DNMT3A p.E423* & \multirow[t]{3}{*}{ Died of relapse after BMT } \\
\hline & & & & & IDH2 p.R140Q & \\
\hline & & & & & STAG2 p.R259* & \\
\hline \multirow[t]{2}{*}{7} & \multirow[t]{2}{*}{$F / 66$} & \multirow[t]{2}{*}{ AML-TMDS } & \multirow[t]{2}{*}{ Normal } & \multirow[t]{2}{*}{ NPM1+, FLT3-ITD-TKD+ } & FLT3 p.D835Y & \multirow[t]{2}{*}{ Died of CNS disease } \\
\hline & & & & & NPM1 p.W288Cfs*12 & \\
\hline 8 & $F / 49$ & $\begin{array}{l}\text { Refractory } \\
\text { AML }\end{array}$ & Not done & FLT3- & SF3B1 p.K666E & Died \\
\hline 9 & $F / 35$ & M2 & $\operatorname{inv}(16)$ & $\mathrm{KIT}+$ & KIT p.D816V & Alive in CR after BMT \\
\hline 10 & $F / 44$ & M4 & Normal & NPM+, FLT3- & NPM1 p.W288Cfs*12 & Alive in $C R$ \\
\hline 11 & $\mathrm{~F} / 82$ & M5a & No growth & FLT3- & Negative & Died \\
\hline 12 & $F / 25$ & M2 & No growth & AML1-ETO-, NPM1-, FLT3-, KIT- & Negative & Alive in CR \\
\hline 13 & $F / 76$ & $\begin{array}{l}\text { AML } \\
\text { transformed } \\
\text { from MDS }\end{array}$ & No growth & FLT3-, del(5q) FISH-, BCR-ABL1- & Negative & Died \\
\hline \multirow[t]{4}{*}{14} & \multirow[t]{4}{*}{$\mathrm{F} / 52$} & \multirow[t]{4}{*}{ M1 } & \multirow[t]{4}{*}{+14} & \multirow[t]{4}{*}{ NPM1-, FLT3- } & CSF3R p.T816l & \multirow[t]{4}{*}{ Lost to follow up } \\
\hline & & & & & IKZF1 p.R111 & \\
\hline & & & & & IDH2 p.R140Q & \\
\hline & & & & & STAG2 p.R1012* & \\
\hline 15 & $M / 82$ & M1 & $\begin{array}{l}\operatorname{add}(3 q), \\
\operatorname{add}(7 q),+8\end{array}$ & FLT3-, BCR-ABL- & Negative & Died \\
\hline \multirow[t]{3}{*}{16} & \multirow[t]{3}{*}{$\mathrm{F} / 59$} & \multirow[t]{3}{*}{ M6 } & \multirow[t]{3}{*}{ Poor growth } & \multirow[t]{3}{*}{ NPM1+, FLT3- } & NPM1 p.W288Cfs*12 & \multirow[t]{3}{*}{ Died } \\
\hline & & & & & ETV6 p.Y346 ${ }^{*}$ & \\
\hline & & & & & IDH2 p.R140W & \\
\hline 17 & $\mathrm{~F} / 78$ & M2 & +10 & NPM1-, FLT3-, AML1-ETO-, PML-RARA- & IDH1 p.R132C & $\begin{array}{l}\text { In CR with vidaza and died of } \\
\text { second cancer }\end{array}$ \\
\hline \multirow[t]{2}{*}{18} & \multirow[t]{2}{*}{$M / 61$} & \multirow{2}{*}{$\begin{array}{l}\mathrm{AML} \\
\text { transformed } \\
\text { from CMML }\end{array}$} & Poor growth & FLT3-, del(5q) FISH-, PDGFRB FISH-, BCR- & CSF3R p.T618l & Died \\
\hline & & & & ABL- & CEBPA p.Y $67^{*}$ & \\
\hline 19 & $F / 64$ & AML-NOS ${ }^{b}$ & Normal & NPM1+, FLT3 ITD-TKD+ & NPM1 p.W288Cfs*12 & Died \\
\hline & & & & & FLT3 p.D835Y & \\
\hline & & & & & DNMT3A p.W313* & \\
\hline & & & & & SMC3 C.3105+2 T>C & \\
\hline & & & & & RAD21 p.Q132* & \\
\hline 20 & $M / 54$ & M1 & Normal & NPM1+, FLT3- & NPM1 p.W288Cfs*12 & Alive in $C R$ \\
\hline & & & & & IDH1 p.R132H & \\
\hline & & & & & FLT3 ITD (25 bp) & \\
\hline & & & & & FLT3 ITD (119 bp) & \\
\hline 21 & $M / 44$ & M1 & $\begin{array}{l}\text { Constitutional } \\
\text { inv(9) }\end{array}$ & FLT3-, BCR-ABL- & Negative & $\begin{array}{l}\text { Died. History of HD treated by } \\
\text { BEACOPP }\end{array}$ \\
\hline
\end{tabular}


Table 1 Summary of 50 patients in this study. (Continued)

\begin{tabular}{|c|c|c|c|c|c|c|}
\hline \multirow[t]{3}{*}{22} & \multirow[t]{3}{*}{$F / 44$} & \multirow[t]{3}{*}{ M2 } & \multirow[t]{3}{*}{ Not done } & \multirow[t]{3}{*}{ FLT3 ITD + TKD- } & NPM1 p.W288Cfs*12 & \multirow[t]{3}{*}{ Died } \\
\hline & & & & & RAD21 c.1161 + 2 T > A & \\
\hline & & & & & FLT3 ITD (72 bp) & \\
\hline 23 & $F / 33$ & $\begin{array}{l}\text { M2 therapy- } \\
\text { related }\end{array}$ & Poor growth & NPM1-, FLT3- & Negative & $\begin{array}{l}\text { Died. History of treated breast } \\
\text { cancer }\end{array}$ \\
\hline 24 & $F / 26$ & M3 & No growth & PML-RARA(s)+, FLT3 ITD + TKD- & FLT3 ITD (33 bp) & Alive in $C R$ \\
\hline 25 & $M / 65$ & M5a & +8 & NPM1-, FLT3 ITD + TKD- & FLT3 ITD (54 bp) & Died \\
\hline 26 & $F / 74$ & MO & +22 & CBFB-MYH11- NPM1- FLT3- BCR-ABL1- & Negative & $\begin{array}{l}\text { Alive in } C R \text { and maintained on } \\
\text { monthly vidaza }\end{array}$ \\
\hline 27 & $F / 77$ & AML-TMDS & +8 & Not done & Negative & $\begin{array}{l}\text { Lost to follow up. History of lung } \\
\text { cancer }\end{array}$ \\
\hline 28 & $M / 18$ & M2 & $t(8 ; 21),-Y$ & AML1-ETO+, KIT-, NPM1-, FLT3- & Negative & Alive in CR \\
\hline 29 & $\mathrm{~F} / 61$ & AML-NOS ${ }^{a}$ & Poor growth & $\begin{array}{l}\text { NPM1-, FLT3 ITD-TKD+, BCR-ABL-, AML1- } \\
\text { ETO- }\end{array}$ & FLT3 p.D835Y & Alive in CR \\
\hline \multirow[t]{2}{*}{30} & \multirow[t]{2}{*}{$F / 39$} & \multirow[t]{2}{*}{ M2 } & \multirow[t]{2}{*}{+8} & \multirow[t]{2}{*}{ AML1-ETO-, NPM+, FLT3- } & NPM1 p.W288Cfs*12 & \multirow[t]{2}{*}{ Alive in CR } \\
\hline & & & & & NRAS p.G12D & \\
\hline \multirow[t]{3}{*}{31} & \multirow[t]{3}{*}{$M / 70$} & \multirow[t]{3}{*}{ M1 } & \multirow[t]{3}{*}{ Normal } & \multirow{2}{*}{$\begin{array}{l}\text { NPM-, FLT3-, PDGFRB FISH-, FIP1L1- } \\
\text { PDGFRA-, BCR-ABL-, }\end{array}$} & TET2 p.S1848* & \multirow{3}{*}{$\begin{array}{l}\text { NR to vidaza. Further treatment in } \\
\text { Mainland China }\end{array}$} \\
\hline & & & & & TET2 p.G1152E & \\
\hline & & & & JAK2 V617F weak+ & $\begin{array}{l}\text { JAK2 p.V617F (VAF } 4.1 \% \text {, } \\
\text { below original reportable } \\
\text { threshold) }\end{array}$ & \\
\hline \multirow[t]{4}{*}{32} & \multirow[t]{4}{*}{$\mathrm{F} / 80$} & \multirow[t]{4}{*}{ M2 } & \multirow[t]{4}{*}{$\operatorname{del}(5 q),+8$} & \multirow[t]{4}{*}{ Not done } & IDH1 p.R132C & \multirow[t]{4}{*}{ Alive and on vidaza } \\
\hline & & & & & TET2 p.K693Nfs*18 & \\
\hline & & & & & TP53 p.R249S & \\
\hline & & & & & ASXL1 p.W960* & \\
\hline \multirow[t]{2}{*}{33} & \multirow[t]{2}{*}{$F / 68$} & \multirow[t]{2}{*}{ M4 } & Normal & FLT3- & NPM1 p.W288Cfs*12 & Difficult CR and on vidaza \\
\hline & & & & & TET2 p.L346Rfs*2 & \\
\hline 34 & $F / 24$ & M2 & $\mathrm{t}(8 ; 21)$ & $\begin{array}{l}\text { AML1-ETO+, BCR-ABL-, PML-RARA-, } \\
\text { NPM1-, FLT3- }\end{array}$ & Negative & Alive in CR \\
\hline 35 & $M / 60$ & M5a & +8 & Not done & NPM1 p.W288Cfs*12 & Alive on treatment \\
\hline & & & & & PTPN11 p.G503A & \\
\hline & & & & & ASXL1 p.G646Wfs*12 & \\
\hline 36 & $\mathrm{~F} / 62$ & M1 & Normal & AML1-ETO-, BCR-ABL1-, PML-RARA-, NPM1 & NPM1 p.W288Cfs*12 & CR for 1 year. Relapsed on \\
\hline & & & & $\begin{array}{l}\text { +, FLT3 ITD+ in cis with } 3 \text { bp deletion } \\
\text { (c.1739_1741delAGG) }\end{array}$ & $\begin{array}{l}\text { FLT3 p.Q580_V581delinsL } \\
\text { (c.1739_1741delAGG) }\end{array}$ & \\
\hline & & & & & FLT3 ITD (54 bp) & \\
\hline & & & & & FLT3 ITD (63 bp) & \\
\hline & & & & & IDH2 p.R140Q & \\
\hline 37 & $F / 46$ & M4 & Near- & AML-ETO-, BCR-ABL1-, & FLT3 p.D835Y & Responded to sorafenib and $\mathrm{HHT}$. \\
\hline & & & tetraploıd & FLT3 ITD+ (30bp), FLT3 TKD+ & FLT3 ITD (30 bp) & $\begin{array}{l}\text { Received BMT from sibling donor } \\
\text { and on treatment }\end{array}$ \\
\hline 38 & M/66 & AML-TMDS & Complex $>3$ & FLT3-, BCR-ABL1-, JAK2 V617F- & DNMT3A p.M801Nfs*11 & Alive on vidaza. History of lung \\
\hline & & & & & TP53 p.R175H & cancer \\
\hline 39 & $F / 62$ & $\begin{array}{l}\text { AML-M5 } \\
\text { post-BMT } \\
\text { relapse }\end{array}$ & $\begin{array}{l}\text { Complex }>3 \\
\text { abnormalities }\end{array}$ & Not done & TP53 p.Y220C & Died \\
\hline 40 & $M / 62$ & M5a & Normal & NPM1+, FLT3-, MLL FISH- & NPM1 p.W288Cfs*12 & Died \\
\hline & & from MDS & & & CBL c.1096-1G > T & \\
\hline 41 & $F / 78$ & AML-TMDS & $\operatorname{add}(21 \mathrm{q})$ & FLT3 ITD + TKD- & FLT3 ITD (30bp) & Died \\
\hline
\end{tabular}


Table 1 Summary of 50 patients in this study. (Continued)

\begin{tabular}{|c|c|c|c|c|c|c|}
\hline 42 & $M / 88$ & AML-NOS ${ }^{*}$ & Not done & Not done & IDH2 p.R140Q & Alive on palliative care \\
\hline 43 & $M / 63$ & M2 & Normal & Not done & IDH2 p.R172K & Alive on vidaza \\
\hline \multirow[t]{3}{*}{44} & \multirow[t]{3}{*}{$\mathrm{F} / 60$} & \multirow{3}{*}{$\begin{array}{l}\text { M2 and } \\
\text { bone } \\
\text { marrow } \\
\text { fibrosis }\end{array}$} & \multirow[t]{3}{*}{ Normal } & \multirow{3}{*}{$\begin{array}{l}\text { 5/7/del(20q) FISH-, BCR-ABL-, FLT3-, JAK2 } \\
\text { V617F/CALR/MPL- }\end{array}$} & DNMT3A c. $855+1 G>T$ & \multirow[t]{3}{*}{ Alive on treatment } \\
\hline & & & & & NPM1 p.W288Cfs*12 & \\
\hline & & & & & IDH2 p.R140Q & \\
\hline 45 & $F / 28$ & M4EO & $\operatorname{inv}(16)$ & CBFB FISH+, CBFB-MYH11 PCR+ & Negative & Alive in $C R$ \\
\hline 46 & $F / 84$ & M4Eo & Not done & CBFB-MYH11 PCR+, FLT3-, NPM1-, KIT- & Negative & Died \\
\hline \multirow[t]{4}{*}{47} & \multirow[t]{4}{*}{$M / 51$} & \multirow{4}{*}{$\begin{array}{l}\text { Atypical } \\
\text { CML }\end{array}$} & \multirow[t]{4}{*}{ Normal } & \multirow{4}{*}{$\begin{array}{l}\text { CSF3R-, BCR-ABL-, JAK2 V617F-, PDGFRB } \\
\text { FISH-, PDGFRA FISH- }\end{array}$} & $\mathrm{EZH} 2$ c.1852-2A > G & \multirow[t]{4}{*}{ Died } \\
\hline & & & & & SETBP1 p.D868N & \\
\hline & & & & & ASXL1 p.G646Wfs*12 & \\
\hline & & & & & ZRSR2 p.Q103* & \\
\hline 48 & $\mathrm{~F} / 53$ & M2 & $\mathrm{t}(8 ; 21)$ & KIT- & SMC3 p.S674_R675insL & Alive in $C R$ \\
\hline 49 & $M / 65$ & $\begin{array}{l}\text { High-grade } \\
\text { MDS }\end{array}$ & Normal & Not done & ASXL1 p.G646Wfs*12 & Alive on vidaza + eltrombopag trial \\
\hline \multirow[t]{2}{*}{50} & \multirow[t]{2}{*}{$M / 51$} & \multirow[t]{2}{*}{ M2 } & \multirow[t]{2}{*}{ Normal } & \multirow[t]{2}{*}{ NPM1-, FLT3-, AML1-ETO- } & CEBPA p.R343Afs*79 & \multirow[t]{2}{*}{ Alive on treatment } \\
\hline & & & & & CEBPA p.K313dup & \\
\hline
\end{tabular}

CR complete remission, NR non-remission, FISH fluorescence in-situ hybridization, vidaza 5-azacytadine, HHT homoharringtonine, HD Hodgkin lymphoma, $P M L-R A R A(s)$ short isoform of fusion transcript from PML bcr3 breakpoint, VAF variant allele frequency, FLT3 testing included detection of both ITD and TKD *Diagnosis on PB only

${ }^{a}$ Aparticulate aspirate and diagnosis by immunophenotyping only

${ }^{b}$ Diagnosis in another hospital

notwithstanding, TP53 mutations were found in three out of four patients (75\%) with complex and unfavorable cytogenetics. The mutational spectrum of the cytogenetic unfavorable group was rather different from that of the cytogenetic normal or intermediate group in our patient cohort. Interestingly, both ASXL1 p.G646Wfs"12 and NPM1 p.W288Cfs*12 mutations were detected in the initial sample of patient 35 but not detected in the post-treatment sample. Although whether the two mutations occurred in the same or independent clones is unknown, they represented one of the first documented exceptions to mutual exclusion nature of $A S X L 1$ and NPM1 mutations in AML [32].

\section{Discussion}

While infrequent in CN-AML, mutations affected TP53 is associated with a complex karyotype [33], as confirmed by our patient cohort. This may define a distinct subgroup of AML that displays primary resistance to therapy and a very dismal prognosis $[34,35]$. It is unclear whether TP53 mutations cause and promote increasing cytogenetic instability, or whether these are secondary mutations occurring after the onset of chromosomal instability. One study showed that a subset of patients with complex karyotype did not have TP53 mutations, whilst all TP53-mutated AML were found in complex karyotype, suggesting that complex karyotype preceded TP53 mutations [36]. Also most mutations of TP53 were associated with del(17p) [36], supporting the contention that TP53 mutations were the second hit in leukemogenesis. More recently, TP53 mutations associated with therapy-related AML were shown to be present before the exposure to chemotherapy, suggesting that a pre-leukemic clone harboring TP53 mutation gained survival advantage after chemotherapy, rather than induced by chemotherapy [37]. Hence TP53mutated AML and therapy-related AML may have more in common than previously recognized.

Detection of FLT3 ITD is an important test for CNAML due to its impact on prognosis and treatment [38]. Highly variable length, allelic burden and number of the ITD mutations were observed [39]. These characteristics pose a challenge in detection by nextgeneration sequencing, specifically not the sequencing process per se, but the bioinformatic analysis of the short sequences obtained $(<300$ bp). Pindel was shown to be the state-of-the-art ITD variant caller, particularly its detection of ITD alleles with length up to 185 bp [29]. However, our study showed that Pindel detected 3 ITD alleles of length 33 bp (patient 24) to $72 \mathrm{bp}$ (patient 22) but not 4 ITD alleles of length 30 to $189 \mathrm{bp}$. Similarly, a recent study showed that Pindel detected 14 ITD alleles of length up to approximately $60 \mathrm{bp}$ but not 3 ITD alleles of length approximately 50, 90 and $110 \mathrm{bp}$ [40].

By comprehensive evaluation based on 40401 simulated ITD alleles with length up to $201 \mathrm{bp}$, we demonstrated that detection from amplicon-based NGS data was dependent on relative ITD position within amplicon, in addition to ITD length. Other laboratories may also 


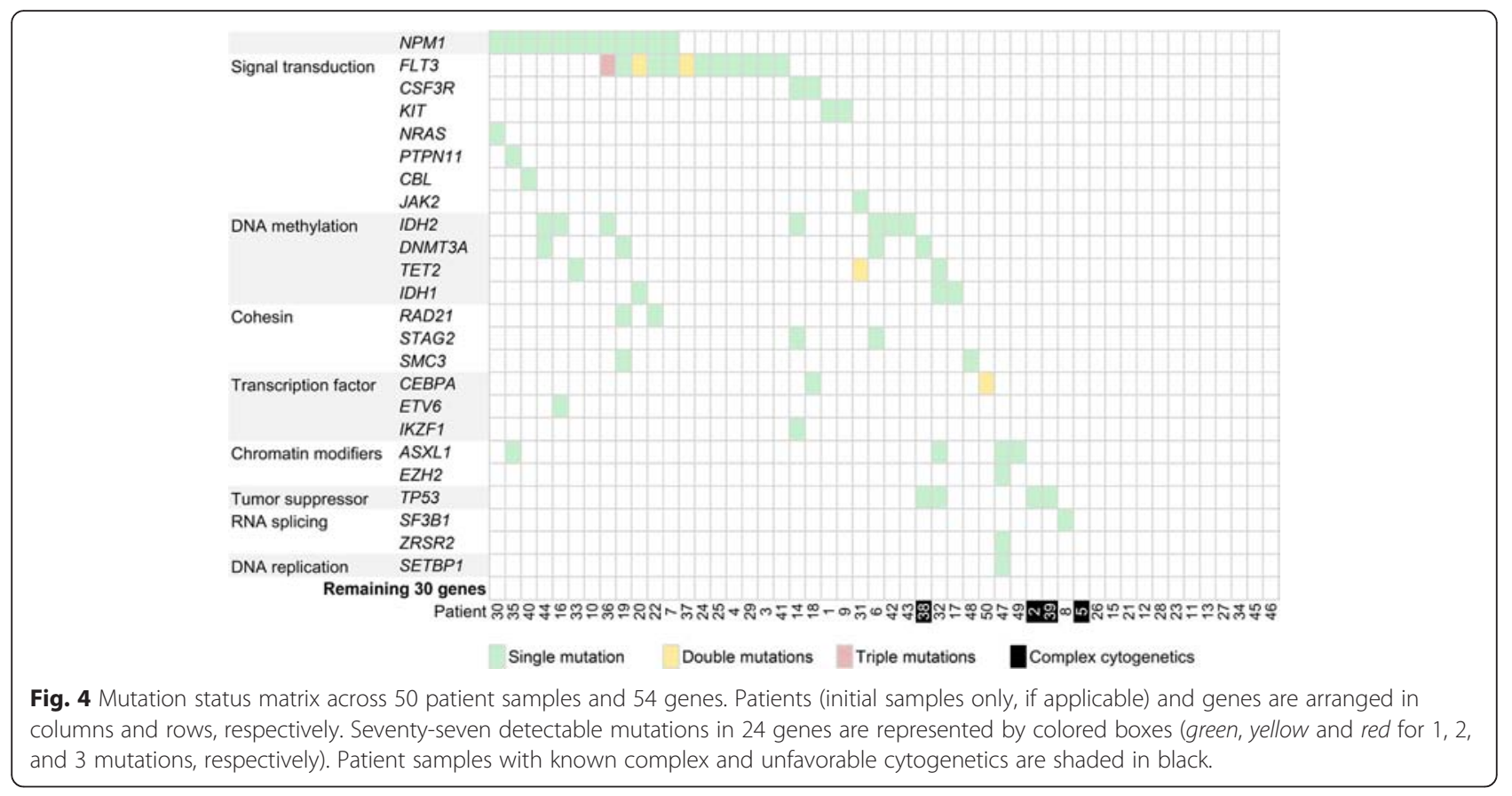

use the simulator ITDsim to evaluate the performance of their own bioinformatics pipeline. We also developed a novel detection algorithm ITDseek because recently developed tools including BreaKmer [41] and Genomon ITDetector [42] were developed for sequencing library prepared with random DNA fragmentation and similar to Pindel. Amplicon Indel Hunter [43] was developed for amplicon-based sequencing data but the actual implementation was not available for parallel evaluation. ITDseek detected most false negatives (97\% of simulated samples) of Pindel, GATK and Samtools. For the actual samples in this study, ITDseek detected ITD alleles up to 189 bp missed by Pindel. ITDseek was designed to process the de facto standard BAM alignment file with minimal computation time $(<20 \mathrm{~s}$ for a whole MiSeq run by $8 \mathrm{CPU}$ cores) and is expected to be easily incorporated in various bioinformatics pipelines of other laboratories.

\section{Conclusions}

We show that gene panel testing by NGS approach in a diagnostic molecular pathology laboratory allows sensitive and accurate detection of actionable AML gene mutations to individualize patient management. The diagnostic approach to AML is facing a paradigm shift in the genomics era [44]. As more targeted therapy become available, the greater is the clinical demand for comprehensive molecular profiling. The results of genomic study hold promise for better understanding disease pathogenesis and classification, refining prognostic stratification and uncovering new drug targets. Further studies should focus on the clinical utility of the genomics to document whether this approach translated into improvements in AML patient outcome and survival [45]. The novel algorithm ITDseek presented in this paper improves the detection of FLT3-ITD in the laboratory setting of amplicon-based next-generation sequencing.

\section{Competing interests}

The authors declare that they have no competing interests.

\section{Authors' contributions}

CHA developed the bioinformatics algorithm and wrote the paper. ESKM and TLC conceived the study. AW and ESKM collected samples and curated the information on diagnosis, conventional testing results and clinical outcome. AW and DNH performed the experiments. CHA, TLC and ESKM analyzed the data. All authors read and approved the final manuscript.

\section{Acknowledgements}

The authors thank all clinicians who provided the patient samples for study and the information on clinical outcome.

Received: 18 December 2015 Accepted: 14 January 2016

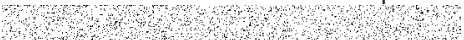

\section{References}

1. Grimwade D, Hills RK, Moorman AV, Walker H, Chatters S, Goldstone AH, et al. Refinement of cytogenetic classification in acute myeloid leukemia: determination of prognostic significance of rare recurring chromosomal abnormalities among 5876 younger adult patients treated in the United Kingdom Medical Research Council trials. Blood. 2010;116(3):354-65.

2. Renneville A, Roumier C, Biggio V, Nibourel O, Boissel N, Fenaux P, et al. Cooperating gene mutations in acute myeloid leukemia: a review of the literature. Leukemia. 2008;22(5):915-31.

3. Dohner H, Estey EH, Amadori S, Appelbaum FR, Buchner T, Burnett AK, et al. Diagnosis and management of acute myeloid leukemia in adults: recommendations from an international expert panel, on behalf of the European LeukemiaNet. Blood. 2010;115(3):453-74.

4. Taskesen E, Bullinger L, Corbacioglu A, Sanders MA, Erpelinck CA, Wouters $B J$, et al. Prognostic impact, concurrent genetic mutations, and gene expression features of AML with CEBPA mutations in a cohort of 1182 
cytogenetically normal AML patients: further evidence for CEBPA double mutant AML as a distinctive disease entity. Blood. 2011;117(8):2469-75.

5. Wouters BJ, Lowenberg B, Erpelinck-Verschueren CA, van Putten WL, Valk PJ, Delwel R. Double CEBPA mutations, but not single CEBPA mutations, define a subgroup of acute myeloid leukemia with a distinctive gene expression profile that is uniquely associated with a favorable outcome. Blood. 2009; 113(13):3088-91.

6. Dufour A, Schneider F, Metzeler KH, Hoster E, Schneider S, Zellmeier E, et al. Acute myeloid leukemia with biallelic CEBPA gene mutations and normal karyotype represents a distinct genetic entity associated with a favorable clinical outcome. J Clin Oncol. 2010;28(4):570-7.

7. Green CL, Koo KK, Hills RK, Burnett AK, Linch DC, Gale RE. Prognostic significance of CEBPA mutations in a large cohort of younger adult patients with acute myeloid leukemia: impact of double CEBPA mutations and the interaction with FLT3 and NPM1 mutations. J Clin Oncol. 2010;28(16):2739-47.

8. O'Donnell MR, Abboud CN, Altman J, Appelbaum FR, Arber DA, Attar E, et al. Acute myeloid leukemia. J Natl Compr Canc Netw. 2012;10(8):984-1021.

9. Mardis ER, Ding L, Dooling DJ, Larson DE, McLellan MD, Chen K, et al. Recurring mutations found by sequencing an acute myeloid leukemia genome. N Engl J Med. 2009;361(11):1058-66.

10. Cancer Genome Atlas Research Network. Genomic and epigenomic landscapes of adult de novo acute myeloid leukemia. N Engl J Med. 2013;368(22):2059-74.

11. Walter MJ, Shen D, Ding L, Shao J, Koboldt DC, Chen K, et al. Clonal architecture of secondary acute myeloid leukemia. N Engl J Med. 2012; 366(12):1090-8.

12. Patel JP, Levine RL. How do novel molecular genetic markers influence treatment decisions in acute myeloid leukemia? Hematology Am Soc Hematol Educ Program. 2012;2012:28-34.

13. Chung SS. Genetic mutations in acute myeloid leukemia that influence clinical decisions. Curr Opin Hematol. 2014;21(2):87-94

14. Schlenk RF, Dohner K, Krauter J, Frohling S, Corbacioglu A, Bullinger L, et al. Mutations and treatment outcome in cytogenetically normal acute myeloid leukemia. N Engl J Med. 2008;358(18):1909-18.

15. Patel JP, Gonen M, Figueroa ME, Fernandez H, Sun Z, Racevskis J, et al. Prognostic relevance of integrated genetic profiling in acute myeloid leukemia. N Engl J Med. 2012:366(12):1079-89.

16. Metzeler KH, Walker A, Geyer S, Garzon R, Klisovic RB, Bloomfield CD, et al. DNMT3A mutations and response to the hypomethylating agent decitabine in acute myeloid leukemia. Leukemia. 2012;26(5):1106-7.

17. Voso MT, Santini V, Fabiani E, Fianchi L, Criscuolo M, Falconi G, et al. Why methylation is not a marker predictive of response to hypomethylating agents. Haematologica. 2014;99(4):613-9.

18. Cortes JE, Kantarjian H, Foran JM, Ghirdaladze D, Zodelava M, Borthakur G, et al. Phase I study of quizartinib administered daily to patients with relapsed or refractory acute myeloid leukemia irrespective of FMS-like tyrosine kinase 3-internal tandem duplication status. J Clin Oncol. 2013; 31(29):3681-7.

19. Wang F, Travins J, DeLaBarre B, Penard-Lacronique V, Schalm S, Hansen E, et al. Targeted inhibition of mutant IDH2 in leukemia cells induces cellular differentiation. Science. 2013;340(6132):622-6.

20. Li H. Toward better understanding of artifacts in variant calling from highcoverage samples. Bioinformatics. 2014;30(20):2843-51.

21. Li H, Handsaker B, Wysoker A, Fennell T, Ruan J, Homer N, et al. The sequence alignment/map format and samtools. Bioinformatics. 2009;25(16): 2078-9.

22. DePristo MA, Banks E, Poplin R, Garimella KV, Maguire JR, Hartl C, et al. A framework for variation discovery and genotyping using next-generation DNA sequencing data. Nat Genet. 2011;43(5):491-8.

23. Koboldt DC, Zhang Q, Larson DE, Shen D, McLellan MD, Lin L, et al. VarScan 2: somatic mutation and copy number alteration discovery in cancer by exome sequencing. Genome Res. 2012;22(3):568-76.

24. Ye K, Schulz MH, Long Q, Apweiler R, Ning Z. Pindel: a pattern growth approach to detect break points of large deletions and medium sized insertions from paired-end short reads. Bioinformatics. 2009;25(21):2865-71.

25. McLaren W, Pritchard B, Rios D, Chen Y, Flicek P, Cunningham F. Deriving the consequences of genomic variants with the Ensembl API and SNP Effect Predictor. Bioinformatics. 2010;26(16):2069-70.

26. Robinson JT, Thorvaldsdottir H, Winckler W, Guttman M, Lander ES, Getz G, et al. Integrative genomics viewer. Nat Biotechnol. 2011;29(1):24-6.

27. Quinlan AR, Hall IM. BEDTools: a flexible suite of utilities for comparing genomic features. Bioinformatics. 2010;26(6):841-2.
28. Stajich JE, Block D, Boulez K, Brenner SE, Chervitz SA, Dagdigian C, et al. The Bioperl toolkit: Perl modules for the life sciences. Genome Res. 2002;12(10):1611-8

29. Spencer DH, Abel HJ, Lockwood CM, Payton JE, Szankasi P, Kelley TW, et al. Detection of FLT3 internal tandem duplication in targeted, short-readlength, next-generation sequencing data. J Mol Diagn. 2013;15(1):81-93.

30. Ohgami RS, Arber DA. The diagnostic and clinical impact of genetics and epigenetics in acute myeloid leukemia. Int J Lab Hematol. 2015;37 Suppl $1: 122-32$

31. Roboz GJ. Epigenetic targeting and personalized approaches for AML. Hematology Am Soc Hematol Educ Program. 2014:2014(1):44-51.

32. Carbuccia N, Trouplin V, Gelsi-Boyer V, Murati A, Rocquain J, Adelaide J, et al. Mutual exclusion of ASXL1 and NPM1 mutations in a series of acute myeloid leukemias. Leukemia. 2010;24(2):469-73.

33. Haferlach C, Dicker F, Herholz H, Schnittger S, Kern W, Haferlach T. Mutations of the TP53 gene in acute myeloid leukemia are strongly associated with a complex aberrant karyotype. Leukemia. 2008;22(8):1539-41.

34. Rucker FG, Schlenk RF, Bullinger L, Kayser S, Teleanu V, Kett H, et al. TP53 alterations in acute myeloid leukemia with complex karyotype correlate with specific copy number alterations, monosomal karyotype, and dismal outcome. Blood. 2012;119(9):2114-21.

35. Kihara $R$, Nagata $Y$, Kiyoi $H$, Kato $T$, Yamamoto $E$, Suzuki $K$, et al. Comprehensive analysis of genetic alterations and their prognostic impacts in adult acute myeloid leukemia patients. Leukemia. 2014;28(8):1586-95.

36. Ohgami RS, Ma L, Merker JD, Gotlib JR, Schrijver I, Zehnder JL, et al. Next-generation sequencing of acute myeloid leukemia identifies the significance of TP53, U2AF1, ASXL1, and TET2 mutations. Mod Pathol. 2015;28(5):706-14

37. Wong TN, Ramsingh G, Young AL, Miller CA, Touma W, Welch JS, et al. Role of TP53 mutations in the origin and evolution of therapy-related acute myeloid leukaemia. Nature. 2015;518(7540):552-5.

38. Leung AY, Man CH, Kwong YL. FLT3 inhibition: a moving and evolving target in acute myeloid leukaemia. Leukemia. 2013:27(2):260-8.

39. Levis M. FLT3 mutations in acute myeloid leukemia: what is the best approach in 2013? Hematology Am Soc Hematol Educ Program. 2013;2013: 220-6.

40. Bolli N, Manes N, McKerrell T, Chi J, Park N, Gundem G, et al. Characterization of gene mutations and copy number changes in acute myeloid leukemia using a rapid target enrichment protocol. Haematologica. 2015;100(2):214-22.

41. Abo RP, Ducar M, Garcia EP, Thorner AR, Rojas-Rudilla V, Lin L, et al. BreaKmer: detection of structural variation in targeted massively parallel sequencing data using kmers. Nucleic Acids Res. 2015;43(3), e19.

42. Chiba K, Shiraishi Y, Nagata Y, Yoshida K, Imoto S, Ogawa S, et al. Genomon ITDetector: a tool for somatic internal tandem duplication detection from cancer genome sequencing data. Bioinformatics. 2015;31(1):116-8.

43. Kadri S, Zhen CJ, Wurst MN, Long BC, Jiang ZF, Wang YL, et al. Amplicon indel hunter is a novel bioinformatics tool to detect large somatic insertion/ deletion mutations in amplicon-based next-generation sequencing data. J Mol Diagn. 2015;17(6):635-43.

44. Bene MC, Grimwade D, Haferlach C, Haferlach T, Zini G. Leukemia diagnosis: today and tomorrow. Eur J Haematol. 2015;95(4):365-73.

45. Wang ML, Bailey NG. Acute myeloid leukemia genetics: risk stratification and implications for therapy. Arch Pathol Lab Med. 2015;139(10):1215-23.

\section{Submit your next manuscript to BioMed Central and we will help you at every step:}

- We accept pre-submission inquiries

- Our selector tool helps you to find the most relevant journal

- We provide round the clock customer support

- Convenient online submission

- Thorough peer review

- Inclusion in PubMed and all major indexing services

- Maximum visibility for your research

Submit your manuscript at www.biomedcentral.com/submit 